\title{
Observation of channeling in bent crystals at the CERN LHC
}

Roberto Rossi*

CERN; Università 'La Sapienza' and INFN Roma I

E-mail: roberto.rossi@cern.ch

W. Scandale, G. Arduini, M. Butcher, F. Cerutti, M. Garattini, S. Gilardoni, A. Lechner, R. Losito, A. Masi, D. Mirarchi, S. Montesano, S. Redaelli, P. Schoofs, G. Smirnov, G. Valentino CERN

D. Breton, L. Burmistrov, V. Chaumat, S. Dubos, J. Maalmi, V. Puill, A. Stocchi

Laboratoire de l'Accelerateur Lineaire (LAL)

E. Bagli, L. Bandiera, G. Germogli, V. Guidi, A. Mazzolari

INFN Sezione di Ferrara and Dipartimento di Fisica e Scienze della Terra, Università di Ferrara

S. Dabagov, F. Murtas

INFN Laboratori Nazionali di Frascati (LNF)

F. Addesa, G. Cavoto, F. Iacoangeli, L. Ludovici, R. Santacesaria, P. Valente

INFN Sezione di Roma

F. Galluccio

INFN Sezione di Napoli

A.G. Afonin, Yu.A. Chesnokov, A.A. Durum, V.A. Maisheev, Yu.E. Sandomirskiy, A.A.

Yanovich

Institute for High Energy Physics in National Research Centre "Kurchatov Institute", IHEP

A.D. Kovalenko, A.M. Taratin

Joint Institute for Nuclear Research, JINR

A.S. Denisov, Yu.A. Gavrikov, Yu.M. Ivanov, L.P. Lapina, L.G. Malyarenko, V.V. Skorobogatov Petersburg Nuclear Physics Institute in National Research Centre "Kurchatov Institute", PNPI

T. James, G. Hall, M. Pesaresi, M. Raymond

Imperial College

The feasibility of crystal-assisted collimation is being investigated for improvements of the LHC collimation system, for possible implementation in the future high luminosity upgrade of the CERN LHC (HL-LHC). Two high-accuracy goniometers, each equipped with one bent silicon crystal, were installed in the betatron cleaning insertion of the LHC in 2014. During dedicated tests in 2015, bent crystals were approached to the circulating beams to test their usage as a first stage in a crystal-based collimation system, both with proton and $\mathrm{Pb}$ ion beams. Tests were performed with protons at injection energy $(450 \mathrm{GeV})$ and at flat top $(6.5 \mathrm{TeV})$, and with ions at injection energy ( $450 \mathrm{Z} \mathrm{GeV}$ ). A reduction of losses immediately downstream of the crystals was observed in optimum channeling orientation, demonstrating for the first time particle channeling at these energies.

38th International Conference on High Energy Physics

3-10 August 2016

Chicago, USA

${ }^{*}$ Speaker. 


\section{Introduction}

High-energy particles interacting with a mono-crystalline material can be trapped by the electromagnetic potential generated by atomic planes in the lattice. Channeling is achieved within a small angular acceptance (critical angle). Bent silicon crystals can steer beam particles to larger angles than the current collimator primary stage. This technology is being considered as a possible path to improve the ion beam collimation for the HL-LHC. Before relying on it, one must demonstrate that channeling works reliably at the LHC design energy and that it improves the performance of the present system. Bent crystals have been installed in the LHC betatron cleaning insertion to address these open points. Results from beam tests in 2015 demonstrated for the first time channeling of $6.5 \mathrm{TeV}$ proton beams [1]. The present LHC collimation system is based on a multi-stage collimation system. Halo beam particles are deflected by means of Multiple Coulomb Scattering (MCS) given by the $60 \mathrm{~cm}$ long primary collimators, then are disposed of by longer secondary collimators and absorbers. A $4 \mathrm{~mm}$ long silicon crystal could give coherent deflections 10 times bigger, reducing at the same time diffractive interaction of beam particles with crystals material. Ideally, one could use a crystal as a primary stage combined with one single absorber to dispose of the halo particles. In this paper, we present the results of the first beam tests that demonstrated crystal channeling at LHC beam energies.

\section{Crystal collimation layout for test in LHC}

Two goniometers, each equipped with a bent crystal were installed on the LHC clockwise Beam 1 (B1), in the horizontal and vertical planes, during the LHC long shutdown in 2014. The goniometers are based on a state-of-the-art piezo-electric technology [2], achieving a resolution of $0.1 \mu \mathrm{rad}$. The goniometers are inserted into a special tank where they are 'parked' during standard operations, shielded from the beam by a special ' $\mathrm{O}$ ' shaped replacement chamber. This chamber hides the goniometers from the circulating beam, making them invisible to the circulating beam

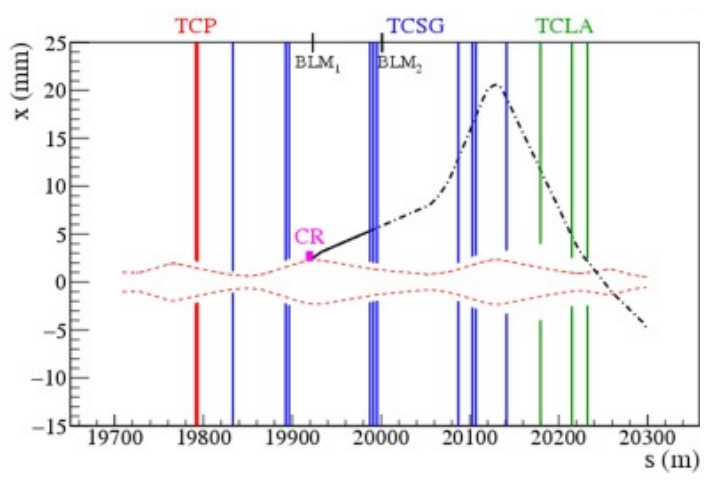

Figure 1: Horizontal projection of the trajectory of a halo particle deflected by the crystal due to channeling at the bend angle $\alpha=65 \mu \mathrm{rad}$ (solid black line) for the maximum momentum of $6.5 \mathrm{TeV}$. In red dashed the beam envelope at $6 \sigma$, vertical lines show the positions of the primary collimators (TCPs - solid red), secondary collimators (TCSGs - solid blue), tertiary debris absorbers (TCLAs - solid green) and the Crystal (purple spot). The positions of BLM1 and BLM2 for measuring the losses in the crystal and in the first horizontal secondary collimator, respectively, are shown schematically. 

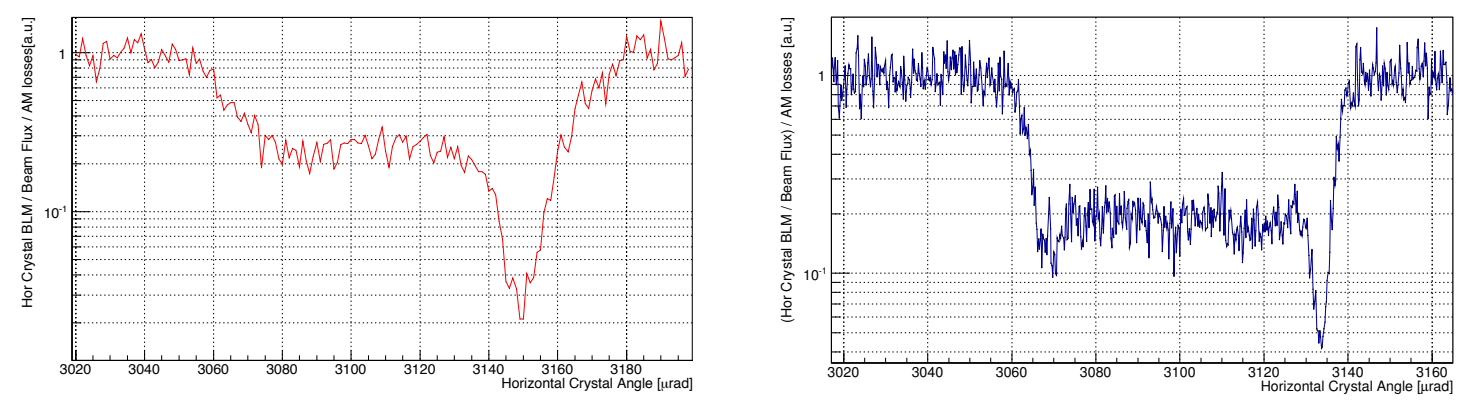

Figure 2: Horizontal crystal angular scans. Beam losses are normalized to the beam flux and to the losses in amorphous orientation. Left. Injection energy scan. Right. Flat Top energy scan.

when they are not intended to be used. Simulation studies [3] have been performed to evaluate the best position and the best crystals properties in order to integrate the crystals in the present collimation system. Those studies were carried aiming to the best crystal collimation performances at LHC flat top (6.5 TeV), as shown in Fig. 1.

\section{Results with protons in 2015 LHC tests}

During 2015, crystals were used in two different Machine Development (MD) sessions. Crystal channeling was established for the first time in LHC at injection energy $(450 \mathrm{GeV})$ during the first session. Channeling is observed as a local reduction of losses when the crystal is parallel to beam envelope (angular scans). This is shown in the left graph of Fig. 2 where the losses immediately downstream of the horizontal crystal are given as a function of the crystal angle. The good results obtained and the excellent orbit and goniometers alignment control allowed the second test to be performed at LHC flat top $(6.5 \mathrm{TeV})$, with injection optics. In this test, channeling was observed for the first time at the LHC record energy as shown in the right graph of Fig. 2. With angular scans it is possible to evaluate the losses reduction between the amorphous orientation (crystal is seen as a random-lattice material) and channeling orientation.

In the flat top angular scan a reduction of the losses by a factor 30 is observed. Other measurements of crystal properties were completed at both energies. For instance, the presence of channeled beam separated from the main beam at the absorber (in this case a secondary collimator)
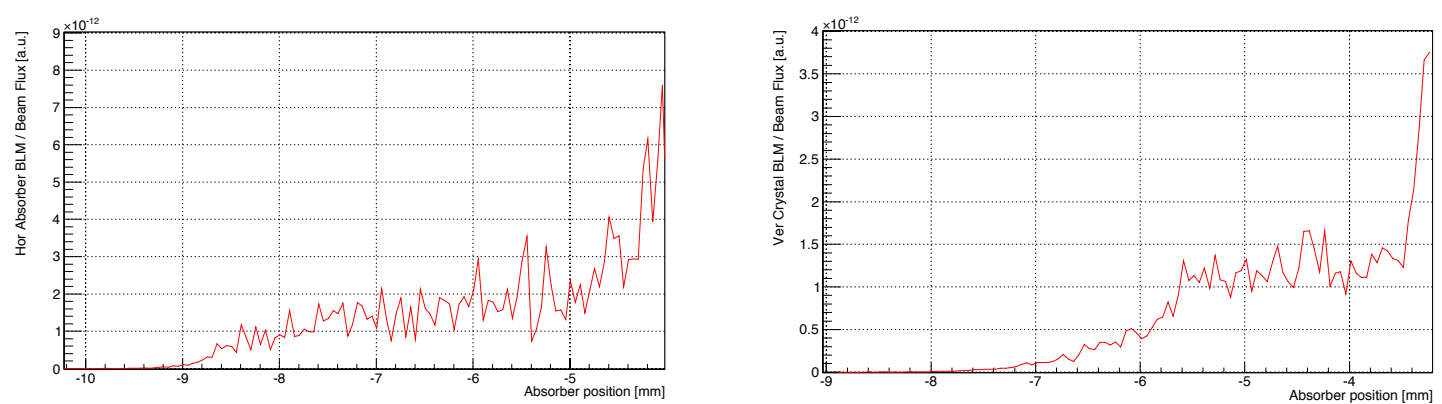

Figure 3: Collimator linear scans with respective crystal in channeling orientation, at injection energy. Beam losses are normalized to the beam flux. Left. Horizontal scan. Right. Vertical scan. 

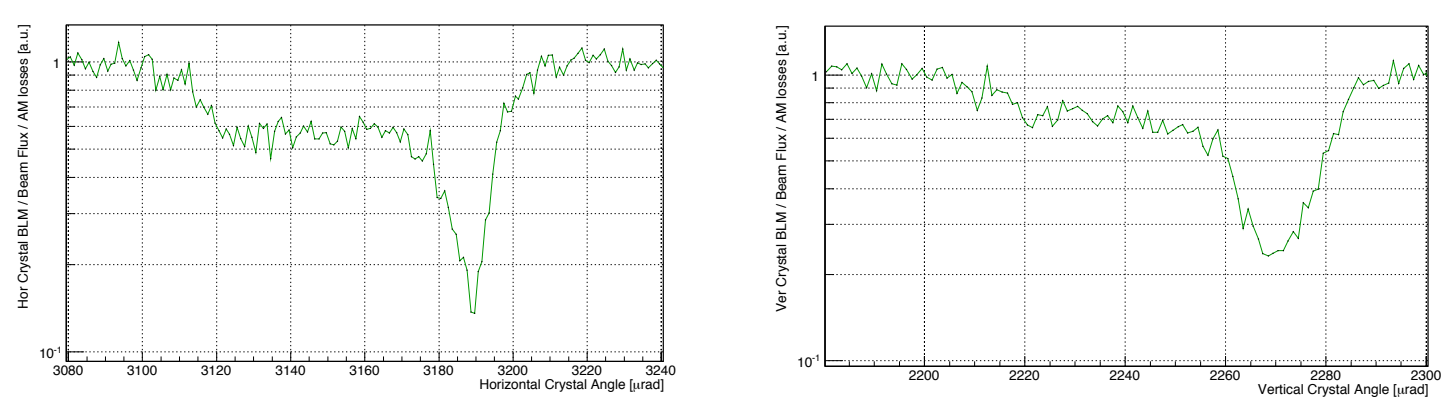

Figure 4: Ions crystal angular scans at injection energy. Beam losses are normalized to the beam flux and to the losses in amorphous orientation. Left. Horizontal crystal. Right. Vertical crystal.

is observed moving the absorber jaw towards the beam core (Fig. 3) with a linear scan. With linear scans is possible to evaluate the crystal bending angle.

\section{Results with ions in 2015 LHC tests}

Results from the proton beam MDs, allowed also to test crystals during the 2015 LHC lead ion run. At injection energy ( $450 \mathrm{Z} \mathrm{GeV})$, ions channeling with bent crystals was observed for the first time in the LHC, both with the horizontal and the vertical crystals.

Evidence of crystal channeling was obtained by monitoring the local losses at the crystal, which, as for proton beams, are reduced when in channeling orientation, as shown in Fig.4. Further confirmation of coherent steering of halo particles through crystal channeling was observed by performing linear scans with the secondary collimator used to catch the channeled halo, indicating the presence of a well-defined halo beam separated from the beam core.

\section{Conclusions}

The results of first beam tests on crystal collimation at the LHC are presented. Channeling was observed at record energies of $6.5 \mathrm{TeV}$ for protons and $450 \mathrm{Z} \mathrm{GeV}$ for $\mathrm{Pb}$ ions. This represents a first important result towards the demonstration of this technology for HL-LHC. Further tests are foreseen in 2016 with the main goal to assess the cleaning performance of a crystal-based collimation system.

\section{References}

[1] W. Scandale et al., Observation of channeling for $6500 \mathrm{GeV/c}$ protons in the crystal assisted collimation setup for LHC, Physics Letters B 758 (2016) 129-133.

[2] M. Butcher, A. Giustiniani, R. Losito, A. Masi, Controller design and verification for a rotational piezo-based actuator for accurate positioning applications in noisy environments, IECON Proceedings (2015), p. 003887.

[3] D. Mirarchi et al., Crystal Collimation for LHC, CERN-THESIS-2015-099.

[4] R. Rossi et al., Crystal Collimation with protons at injection energy, CERN-ACC-NOTE-2016-0035. 